\title{
PD-L1 expression in lung cancer
}

\author{
Chad Tang, Joe Y. Chang \\ Department of Radiation Oncology, MD Anderson Cancer Center, Houston, USA \\ Correspondence to: Chad Tang. Department of Radiation Oncology, MD Anderson Cancer Center, Houston, USA. Email: ctang1@mdanderson.org; \\ Joe Chang. Department of Radiation Oncology, MD Anderson Cancer Center, Houston, USA. Email: jychang@mdanderson.org.
}

Submitted Oct 20, 2016. Accepted for publication Nov 01, 2016.

doi: $10.21037 /$ jtd.2016.11.38

View this article at: http://dx.doi.org/10.21037/jtd.2016.11.38

It is with great interest that we read the recent manuscript written by Dr. Yu and colleagues in the Fournal of Thoracic Oncology (1). In this manuscript Yu et al. describes different aspects of PD-L1 expression both at the protein and mRNA level in two cohorts of small cell lung cancer (SCLC) patients (1). These data are especially timely given the recent publication of Checkmate 032, a phase I/II trial of nivolumab and nivolumab plus ipilimumab in patients with recurrent small-cell lung cancer (2). Their data provides further clarification regarding PD-L1 pattern in SCLC, a cancer that has not been extensively treated with immunotherapy agents, but for which there is significant biologic rationale given the high mutation burden (3).

The impetus for PD-L1 staining is driven by recent nonsmall cell lung cancer (NSCLC) trials that demonstrated associated with endpoints including objective response, progression free survival, and overall survival (4-7). Partially as a result, percent tumor PD-L1 expression level has arisen as an inclusion criteria for the most recent randomized studies comparing PD-1 inhibitors to standard of care chemotherapy as first or second line therapy of metastatic NSCLC $(8,9)$. Further corroborating these results are recent data from KEYNOTE-141 where PD-L1 expression was associated with an overall survival benefit for nivolumab treatment (10). Despite these associations in NSCLC and head and neck cancer, data in other histology have yielded mixed results. Trials in melanoma and renal cell carcinoma have not demonstrated significant associations between patient outcomes and PD-L1 expression (11-13). To explain the heterogeneity of the observed association between PD-L1 expression and clinical response, we feel an often overlooked aspect is technical details of the tumor biopsy and PD-L1 staining. In our experience there are significant differences in PD-L1 staining when scoring different size biopsy samples (unpublished data). We attribute this to the variable spatial expression patterns of surface PD-L1 expression on tumor cells. We have observed that PD-L1 expression occurs in isolated clusters or band-like "expression fronts". When such regions of high PD-L1 density are by chance sampled, higher PD-L1 expression is recorded than if a larger biopsy (or whole tumor sections) were to sample areas of both high and low PD-L1 expression. Furthermore, variations also exist in staining technique, biopsy timing and technique, organ site biopsied, and varying anti-PD-L1 antibodies. It is therefore of particular relevance that $\mathrm{Yu}$ et al. investigates the variability in PD-L1 staining with different antibody clones and PD-L1 expression at the mRNA level (1).

Current interest in treating SCLC with immunotherapy agents stems from the high number of somatic mutations that characterize this cancer (3). The association of somatic mutation with disease response to pembrolizumab was demonstrated in a phase 2 study of patients treated for mismatch repair deficient cancers (14). This study found a high objective response rate of 53\%. Of note, both this trial (14) and others $(15,16)$ observed a significant association between a high somatic mutation load and response. Despite this, the objective response rate of SCLC patients in Checkmate 032 with nivolumab alone was modest (10\%), with tumor responses occurring irrespective of PD-L1 status (2). More encouraging were the objective response rates of patients treated with combination ipilimumab and nivolumab (22\%). It is worth noting that the rate of grade 3-4 toxicity associated with this combination therapy were not as high as those observed in earlier trials treating melanoma with combination immunotherapy (17). To further explore the role of immunotherapy for SCLC, our department is conducting an investigator-initiated phase I study to assess 
the effects of pembrolizumab and radiation in extensive and limited stage disease (NCT02402920). The rationale behind such a treatment paradigm is that radiation releases antigens, providing greater immune system access to the array of somatic mutations inherent in this disease $(18,19)$.

In conclusion, although PD-L1 testing is fast emerging as standard test in to select immunotherapy treatment for NSCLC, whether such a test exhibits utility in SCLC remains to be determined. The analysis conducted by $\mathrm{Yu}$ et al. provides further insight into PD-L1 testing and expression levels for SCLC (1), a valuable addition to the literature especially as data on immunotherapy treatment for SCLC emerges. Finally, we stress that although strong biologic rationale exists for immunotherapy selection based on PD-L1 staining, variability in staining and biopsy samples may produce a level of inter-sample variability that makes these associations difficult to identify.

\section{Acknowledgements}

None.

\section{Footnote}

Provenance: This is an invited Editorial commissioned by the Section Editor Ming-Hui Zhang (Department of Medical Oncology, Harbin Medical University Cancer Hospital, Harbin, China).

Conflicts of Interest: The authors have no conflicts of interest to declare.

Comment on: Yu H, Batenchuk C, Badzio A, et al. PD-L1 Expression by Two Complementary Diagnostic Assays and mRNA In Situ Hybridization in Small Cell Lung Cancer. J Thorac Oncol 2016. [Epub ahead of print].

\section{References}

1. Yu H, Batenchuk C, Badzio A, et al. PD-L1 Expression by Two Complementary Diagnostic Assays and mRNA In Situ Hybridization in Small Cell Lung Cancer. J Thorac Oncol 2016. [Epub ahead of print].

2. Antonia SJ, López-Martin JA, Bendell J, et al. Nivolumab alone and nivolumab plus ipilimumab in recurrent smallcell lung cancer (CheckMate 032): a multicentre, openlabel, phase 1/2 trial. Lancet Oncol 2016;17:883-95.

3. Lawrence MS, Stojanov P, Polak P, et al. Mutational heterogeneity in cancer and the search for new cancer- associated genes. Nature 2013;499:214-8.

4. Borghaei H, Paz-Ares L, Horn L, et al. Nivolumab versus Docetaxel in Advanced Nonsquamous Non-Small-Cell Lung Cancer. N Engl J Med 2015;373:1627-39.

5. Garon EB, Rizvi NA, Hui R, et al. Pembrolizumab for the treatment of non-small-cell lung cancer. N Engl J Med 2015;372:2018-28.

6. Herbst RS, Soria JC, Kowanetz M, et al. Predictive correlates of response to the anti-PD-L1 antibody MPDL3280A in cancer patients. Nature 2014;515:563-7.

7. Fehrenbacher L, Spira A, Ballinger M, et al. Atezolizumab versus docetaxel for patients with previously treated non-small-cell lung cancer (POPLAR): a multicentre, open-label, phase 2 randomised controlled trial. Lancet 2016;387:1837-46.

8. Reck M, Rodríguez-Abreu D, Robinson AG, et al. Pembrolizumab versus Chemotherapy for PD-L1-Positive Non-Small-Cell Lung Cancer. N Engl J Med 2016. [Epub ahead of print].

9. Herbst RS, Baas P, Kim DW, et al. Pembrolizumab versus docetaxel for previously treated, PD-L1-positive, advanced non-small-cell lung cancer (KEYNOTE-010): a randomised controlled trial. Lancet 2016;387:1540-50.

10. Ferris RL, Blumenschein G Jr, Fayette J, et al. Nivolumab for Recurrent Squamous-Cell Carcinoma of the Head and Neck. N Engl J Med 2016. [Epub ahead of print].

11. Hamid O, Robert C, Daud A, et al. Safety and tumor responses with lambrolizumab (anti-PD-1) in melanoma. N Engl J Med 2013;369:134-44.

12. Robert C, Schachter J, Long GV, et al. Pembrolizumab versus Ipilimumab in Advanced Melanoma. N Engl J Med 2015;372:2521-32.

13. Motzer RJ, Rini BI, McDermott DF, et al. Nivolumab for Metastatic Renal Cell Carcinoma: Results of a Randomized Phase II Trial. J Clin Oncol 2015;33:1430-7.

14. Le DT, Uram JN, Wang H, et al. PD-1 Blockade in Tumors with Mismatch-Repair Deficiency. N Engl J Med 2015;372:2509-20.

15. Van Allen EM, Miao D, Schilling B, et al. Genomic correlates of response to CTLA-4 blockade in metastatic melanoma. Science 2015;350:207-11.

16. Rizvi NA, Hellmann MD, Snyder A, et al. Cancer immunology. Mutational landscape determines sensitivity to PD-1 blockade in non-small cell lung cancer. Science 2015;348:124-8.

17. Postow MA, Chesney J, Pavlick AC, et al. Nivolumab and ipilimumab versus ipilimumab in untreated melanoma. $\mathrm{N}$ Engl J Med 2015;372:2006-17. 
18. Tang C, Wang X, Soh $\mathrm{H}$, et al. Combining radiation and immunotherapy: a new systemic therapy for solid tumors? Cancer Immunol Res 2014;2:831-8.

19. Tang C, Welsh JW, de Groot P, et al. Ipilimumab with

Cite this article as: Tang C, Chang JY. PD-L1 expression in lung cancer. J Thorac Dis 2016;8(11):3053-3055. doi: 10.21037/ jtd.2016.11.38 stereotactic ablative radiation therapy: Phase I results and immunologic correlates from peripheral T-cells. Clin Cancer Res 2016. [Epub ahead of print]. 